\title{
Gastric cancer with high risk of intraperitoneal progression: clinical course and current treatments
}

\author{
ROMAN YAREMA
}

Department of Oncology and Medical Radiology, Danylo Halytsky Lviv National Medical University, Pekarska 69, 79010, Lviv, Ukraine

\section{ARTICLE INFO \\ Received 27 October 2017 \\ Accepted 29 December 2017}

\section{Keywords:}

gastric cancer, |

metachronous peritoneal

carcinomatosis,

hyperthermic intraperitoneal

chemotherapy.

\begin{abstract}
Locally advanced gastric cancer with a high risk of intraperitoneal progression is characterized by poor prognosis. After radical surgery, most patients die during the first two years post-operation as a result of disease progression. The prevailing type of progression and the leading cause of death in patients with gastric cancer is implantation metastasis.

The main risk factors for peritoneal carcinomatosis in such patients include: gastric tumor invasion into serosa, the presence of tumor cells in peritoneal washings, the largeness of the tumor as accompanied by extensive serous lesions, infiltrative type of tumor growth, histological variants of gastric cancer prone to implantation metastasis and metastatic lesions in regional lymph nodes. Systemic chemotherapy does not provide effective eradication of subclinical peritoneal carcinomatosis in patients with locally advanced gastric cancer.

The vast majority of patients who suffer from locally advanced gastric cancer and run a high risk of implantation metastasis are characterized by subclinical peritoneal dissemination at primary diagnosis, which means a rapidly fatal prognosis for such patients. In recent years, however, the paradigm of treatment of locally advanced gastric cancer has changed: a combination of surgery and adjuvant hyperthermic intraperitoneal chemotherapy is used increasingly, and presents an alternative to the previously accepted surgery only approach. It is also likely to increase the survival rate.
\end{abstract}

\section{INTRODUCTION}

Locally advanced gastric cancer (GC) (T3-4 N+ M0) is characterized by a poor prognosis [1]. Therefore, even after radical surgery, most patients die during the first two years post-operation as a result of disease progression [2]. The prevailing type of $\mathrm{GC}$ progression is implantation metastasis $[3,4]$, which probably comes about due to the presence of a microscopic pool of tumor cells in the peritoneum before the time of surgery [5] or because of intraoperative peritoneal dissemination [6]. Of note, metachronous peritoneal carcinomatosis (PC) develops after radical surgery in 50\% of all patients with a tumor invasion of serosa [7]. Peritoneal carcinomatosis is the leading cause of death in GC patients, with an average median survival of 6 months after the diagnosis of intraperitoneal recurrence [8].

The problem of intraperitoneal metachronous progression of GC after surgical treatment has been relevant for gastric surgery since the first surgeries for cancer. Indeed, after the

\footnotetext{
* Corresponding author

e-mail: roman.yarema@ukr.net
}

first gastrectomy (performed by T. Billroth, in 1881), the patient lived for 4 months and died of disease progression; the autopsy showed widespread metastases in the omentum, as well as metastases in the liver [9].

According to current literature data, PC occurs in $38-60 \%$ of all patients after radical surgery for GC $[10,11]$. The crucial risk factors for $\mathrm{PC}$ development in these patients include: tumor invasion into the gastric serosa [12], the presence of tumor cells in peritoneal lavage [13], the largeness of the tumor and the accompaniment of extensive serosa lesions [4,14], infiltrative type of tumor growth [15], histological variants of GC prone to implantation metastasis [16] and metastatic lesions of regional lymph nodes [17].

The depth of tumor invasion in the wall of a cavernous organ is without doubt the critical factor of implantation metastasis. Invasion of the gastric serosa is an independent adverse prognostic factor which determines the possibility of dissemination of tumor cells in the abdomen and the subsequent rapid fatal prognosis $[14,18]$. According to Roviello et al. [17], intraperitoneal recurrence after surgical treatment 
took place in $69 \%$ of all patients with serosa invasion and $\mathrm{GC}$ of the diffuse type. Statistically, categories T4a/4b (serosa invasion/ingrowth into adjacent organs - according to TNM-7th edition 2009 [19]) are likely to correlate with the level of intraperitoneal progression and a sharp decrease in the survival rate [20]. However, even if there was no invasion of the gastric serosa, metachronous PC developed in $37 \%$ of all patients [21]. The mechanisms of intraperitoneal progression in this case may be connected with both tumor cell migration through unaffected layers of the wall of the stomach and their intraoperative dissemination because of the intersection of lymphatic vessels in the course of lymphadenectomy [22]. A clear connection between PC development and radical surgery/depth of invasion of the primary gastric tumor is shown in a study by S. N. Nered [23]: in the case of pT1 tumors, the intraperitoneal recurrence rate was $12.5 \%$, in the case of pT2 $-29.6 \%$ and in the case of ingrowth into serosa, it exponentially increased to $46.2 \%$. In addition, the depth of invasion also affected the length of disease-free period - in the case of pT1, the average life expectancy before PC detection was 54.7 months, whereas in case of pT4a, it was 19.8 months. Metachronous peritoneal carcinomatosis is characterized by the shortest disease-free period in comparison with other ways of GC progression [24].

The presence of free tumor cells in the peritoneal cavity is another independent negative prognostic factor of locally advanced GC [25]. According to the results of peritoneal lavage cytology in patients with $\mathrm{T} 4 \mathrm{a} / 4 \mathrm{~b}$ tumors, cancer cells are found in 10\% [13] - 44\% [26] of all cases. Moreover, according to Bando et al. [25], 5\% of patients without macroscopic serosa invasion had positive peritoneal lavage. It is natural that there is a close connection between the affected area of the gastric serosa and the frequency of positive cytologic findings. Thus, according to Iceguchi et al. [27], if the area of peritoneal lesion is smaller than $10 \mathrm{~cm}^{2}$, free tumor cells are detected in the lavage in $17.3 \%$ of all cases, whereas if it is bigger than $20 \mathrm{~cm}^{2}$, they are detected in $68.5 \%$ of all cases. Kaibara et al. [26] also observed a significant difference in positive peritoneal lavage between patients whose affected area of the serosa is smaller than $10 \mathrm{~cm}^{2}$, and whose that is bigger than $20 \mathrm{~cm}^{2}-22 \%$ and $72 \%$, respectively. Adachi et al. [28] add that the 5-year survival of patients with tumor diameter below and above $10 \mathrm{~cm}$ is $42 \%$ and $14 \%$, respectively. Bando et al. [25] state that a serosa infiltration which is more than $2.5 \mathrm{~cm}$ in diameter is an independent predicative factor of peritoneal $\mathrm{GC}$ recurrence. Other studies have noted that the highest occurrence of metachronous PC is observed in patients with total circular lesion of the stomach $-64.9 \%$ [23]. This situation often accompanies undifferentiated and colloid carcinomas [29].

Extended lymph node dissection at the time of the surgical treatment of GC can contribute to intraperitoneal dissemination of tumor cells, especially in cases of wide lymphogenous spread of the primary tumor [23]. On the other hand, the positive effect of extended lymphadenectomy D2 on survival, which is becoming common today, is eliminated by the rapid development of metachronous PC. Indeed, it has been shown that surgery with D2-lymphadenectomy in cases of $\mathrm{GC}$ with a high risk of implantation metastasis does not increase survival [4].

Thus, the presence of free tumor cells in the peritoneum determines a clear negative prognosis for patients - in one study, none of the patients survived during a 3-year followup period after radical surgery [30]. This result stands in agreement with the study of Bentrem et al. [13] wherein the median overall survival for patients who have positive results of intraoperative peritoneal lavage cytology is 14.8 months, whereas in the case of the negative cytological conclusion it is 98.5 months. Furthermore, La Torre et al. [22] report a median survival of 19 months in patients with positive peritoneal lavage, against 38 months in patients with negative lavage. Therefore, many authors believe that the presence of tumor cells in peritoneal lavage from GC patients is a sign of tumor dissemination, and this situation calls for palliative treatment regardless of the extent of interventions performed [31]. Thus, according to the clinical classification of the Japanese Gastric Cancer Association (JGCA), in contrast to the 2002 version of TNMclassification of the Union for International Cancer Control (UICC), these patients are definitely stratified to stage IV of the disease [32]. Therefore, intraoperative cytological examination of peritoneal lavage is a routine procedure in Japan today. In the $\mathrm{TNM}-7^{\text {th }}$ edition 2009 , the peritoneal lavage category was introduced - positive lavage being viewed as a sign of distant metastases [19].

To assess the degree of risk of implantation dissemination, it is necessary to take into account the histological structure and macroscopic appearance of the primary tumor. In terms of the features of metastasis in patients with locally advanced GC and disease progression after surgery, implantation metastasis usually develops in cases of colloid (65\%), undifferentiated and poorly differentiated (52-54\%) cancers. Well- and moderately-differentiated adenocarcinoma rarely accompanies PC (17.3-27\%), but it hides a high risk of hematogenous metastasis to the liver (47.9-52\%), while at colloid, cancer liver metastases occur only in $9,6 \%$ of cases [33]. The histological grade of GC also affects the time of disease progression, so the shortest disease-free survival (11-14.5 months) is observed in patients with poorly differentiated adenocarcinoma, colloid and undifferentiated cancer [21].

Diffuse or scirrhous GC, according to Lauren's classification, is characterized by a high risk of implantation metastasis $-31 \%$ of all cases were accompanied by PC, whereas with regard to the so-called intestinal or differentiated type, peritoneal dissemination was observed in only $6 \%$ of all patients. At that, no clear difference in the frequency of lymphogenous metastasis was revealed between the two types of GC [28]. The macroscopic appearance of GC also points to the risk of implantation dissemination. Thus, GC type I according to Borrmann's classification (1926) (an exophytic tumor) is accompanied by PC in $6.2 \%$ of all patients with a progressing disease, type II (saucer-shaped cancer) in $23.4 \%$, type III (ulcerative-infiltrative cancer) - in $48.1 \%$ and type IV (diffuse, or infiltrating, cancer) - in $61.4 \%$ of all patients [23].

Thus, in a considerable number of patients, locally advanced GC with a high risk of implantation metastasis 
is accompanied by subclinical peritoneal dissemination at diagnosis, as shown by PC manifestation within the first months or years after treatment. Therefore, it is clear that these patients require combined therapy.

For a long time, hopes of adjuvant chemotherapy of GC had no confirmation in randomized studies [34]. For example, two meta-analyses of randomized studies of previous years showed no increase in survival due to the use of systemic adjuvant chemotherapy in patients with resectable GC $[35,36]$. However, in 2006, the results of MAGIC research [10] were published. Herein, it was evident that doctors had definitely managed to increase the 5-year survival rate in patients who underwent preoperative and postoperative ECF chemotherapy (from $23 \%$ to $36 \%$ ) when compared to a surgery group of patients. This approach is currently the national standard of GC treatment in the UK and in some EU countries. A detailed analysis of the data of this research shows that the percentage of patients with a high risk of implantation metastasis in the study groups is small - only about $20 \%$ of all patients had tumor diameter bigger than $8 \mathrm{~cm}$. In a North-American multicenter study - SWOG 9008 (INT 0116) - patients were randomized for surgery and for combined therapy by fluorouracil/leucovorin postoperative chemotherapy before, during, and after radiation therapy (45 Gy). In the study group, the 5-year survival rate was increased to $15 \%$ of all patients [11]. Of note, while the percentage of patients with T4a tumors in the study group was high (about 60\%), only 64\% managed to complete the combined therapy due to its toxicity. Today, this GC therapy is standard in the US and Canada. In Japan, the national standard of GC treatment is based on the results of the randomized study, ACTS-GC, published by Sacuramoto et al. in 2007, which confirmed the growth of 3-year survival in $10 \%$ of all patients who received systemic adjuvant chemotherapy S1 [37]. In addition, a statistically significant reduction in the incidence of metachronous lymphogenous and peritoneal metastases was received in the study group, but the frequency of implantation metastasis decreased inconsiderably (from $15.8 \%$ in the surgical treatment group, to $11.2 \%$ in the combined therapy group).

In 2007, the results of phase II of a clinical study (the Borrmann JCOG 0210-study) of the use of neoadjuvant chemotherapy S1 + cisplatin in patients with locally advanced GC types 3-4 were published. In this, 3-year survival was achieved in $26 \%$ of all patients [38]. Moreover, the application of the same chemotherapy regimen in patients with seropositive GC allowed a 3-year survival in $43 \%$ of all patients [39], however, serosa invasion was pathomorphologically confirmed in only $61 \%$ of the studied patients.

Thus, systemic chemotherapy does not provide effective eradication of subclinical PC in patients with locally advanced GC with a high risk of implantation progression. Potential factors causing relatively poor results of combined therapy in such patients include relatively low sensitivity of GC to chemotherapy and the supply of inadequate doses of drugs directly to tumor microdeposits in the peritoneum [40].

Because gastric cancer is a relatively chemo-resistant tumor, it is now hoped to increase the effectiveness of combined therapy by changing modes of administration of chemotherapeutic agents and by using modulation techniques of chemotherapeutic intervention [41].

The first report on intra-abdominal chemotherapy was published in 1955, by Weisberger et al. [42]. The first report of intraperitoneal chemotherapy for GC was published by Kimura in 1979 [43]. The combination of intraperitoneal administration of chemotherapeutic agents and local hyperthermia was first applied by the American surgeon Spratt et al. in 1980, to treat a pseudomixoma peritonei [44]. Later, this treatment option was called hyperthermic intraperitoneal chemotherapy (HIPEC).

Pharmacokinetic studies show that at intraperitoneal administration of cytostatic agents of high molecular weight (mitomycin, cisplatin, doxorubicin etc.), the gradient of their concentration remains high for a long time due to the existence of the peritoneal-plasma barrier. This situation allows for the creating of a high concentration of chemotherapeutic agents in the peritoneum with low systemic toxicity [45].

One of the promising directions of using hyperthermic intraperitoneal chemotherapy in oncology is its use in the adjuvant mode for patients with locally advanced GC with a high risk of implantation progression [46]. Thus, Fujimoto et al. [47] present the results of a combined therapy of 71 GC patients with a high risk of implantation metastasis with the use of HIPEC (mitomycin, $90 \mathrm{~min}$ at $44.0-45.0^{\circ} \mathrm{C}$ ), which allowed achieving 2-, 4 - and 8-year survival in the study group at the level of $88 \%, 76 \%$ and $62 \%$, whereas in the control group, survival rates were $77 \%, 58 \%$ and $49 \%$, respectively.

The results of a randomized controlled study of extended gastrectomy combined with HIPEC in an adjuvant mode (mitomycin $30 \mathrm{mg}$ + cisplatin $300 \mathrm{mg}, 90 \mathrm{~min}$ at 42.0$43.0^{\circ} \mathrm{C}$ ) are presented by Yonemura et al. [48]. In the combined therapy group, the 5-year survival rate reached $61 \%$, as against $42 \%$ in the surgery group. The authors conclude that HIPEC is effective in the prevention of PC progression after GC radical surgery.

Kim et al. [49] find that after interventions with D1 perigastric lymphadenectomy, the rate of intraperitoneal recurrence is $33.3 \%$, while after lymph node dissection D2, it increases to $46.7 \%$. This level of increase is attributed to the intraperitoneal dissemination of tumor cells as a result of the intersection of a large number of lymphatic ducts. However, not a single case of metachronous PC development and the highest follow-up indices were recorded after interventions with lymph node dissection D2 combined with HIPEC. Indeed, GC intraperitoneal recurrence rate in cases of using HIPEC after radical surgery ranges from $0-5 \%$ to $39 \%$, whereas in the control group it is $48-59 \%$, according to different authors $[49,47]$.

In 2004, the Cochrane Collaboration performed a metaanalysis of 11 prospective randomized studies of the effectiveness of adjuvant intraperitoneal chemotherapy use in radically operated patients suffering from locally advanced GC [50]. The total number of study patients was 1,161. This meta-analysis statistically showed that combined therapy was more effective than that used in the control group. The best results of general and disease-free survival were obtained in cases where HIPEC was used. 
In 2007, results of another meta-analysis of randomized studies of adjuvant intraperitoneal chemotherapy of resectable GS were published. It presents a cooperative analysis of the results of treatment of over 1.6 thousand patients within 13 randomized studies which have the highest level of evidence and were selected from 106 reports found in literature [51]. The results of this meta-analysis show that HIPEC alone or in combination with normothermic intraperitoneal chemotherapy is associated with a statistically significant survival rate growth.

In conclusion, the analysis of literature data shows that the vast majority of patients who suffer from locally advanced GC and run a high risk of implantation metastasis are characterized by subclinical peritoneal dissemination at primary diagnosis, which means a rapidly fatal prognosis for such patients. In recent years, however, the paradigm of treatment of locally advanced GC has changed: a combination of surgery and adjuvant HIPEC is used increasingly, and this presents an alternative to the previously accepted exclusively surgical method. It is also likely to increase the survival rate.

\section{REFERENCES}

1. Siewert JR, Bottcher K, Stein HJ et al. Relevant prognostic factors in gastric cancer: ten-year results of the German Gastric Cancer Study. Ann Surg. 1998;228(4):449-461.

2. Lim L, Michael M, Mann GB, Leong T. Adjuvant therapy in gastric cancer. J Clin Oncol. 2005;23(25):6220-6232.

3. Yoo $\mathrm{CH}, \mathrm{Noh} \mathrm{SH}$, Shin DW et al. Recurrence following curative resection for gastric carcinoma. Br J Surg. 2000;87(2):236-242.

4. Nered SN, Klimenkov AA. Surgical treatment of gastric cancer with a high risk of implantation metastasis. Questions Oncology. 2005;51(1):75-80.

5. Koga S, Kaibara N, Itsuka $\mathrm{Y}$ et al. Prognostic significance of intraperitoneal free cancer cells in gastric cancer patients. J Cancer Res Clin Oncol. 1984;108:236-238.

6. Van den Tole P, Van Rossen E, Van Eijck C et al. Reduction of peritoneal trauma by using non surgical gauze leads to less implantation metastases of spilled tumour cells. Ann Surg. 1998;227(2):242-248.

7. Chen S, Cai MY, Chen YB et al. Serosa-penetration in human T4aN0M0 gastric carcinoma correlates with worse prognosis after D2 gastrectomy. Chin Med J. 2012;125(6):1158-1162.

8. Maekawa S, Saku M, Maehara Y et al. Surgical treatment for advanced gastric cancer. Hepatogastroenterology. 1996;43(7):178-186

9. Arhelger SW. Advances in diagnosis and management of gastric cancer: II. Trends in the management of gastric csncer. CA Cancer J Clin. 1957;7:161-170.

10. Cunningam D, Allum WH, Stenning SP et al. Perioperative chemotherapy versus surgery alone for resectable gastro-esophageal cancer. N Engl J Med. 2006;355(1):11-20.

11. MacDonald JS, Malley SR, Benedetti J et al. Chemoradiotherapy after surgery compared with surgery alone for adenocarcinoma of the stomach or gastroesophageal junction. N Engl J Med. 2001;345(10):725-730

12. Wu CW, Lo Wu SS, Shen $\mathrm{KH}$ et al. Incidence and factors associated with recurrence patterns after intended curative surgery for gastric cancer. World J Surg. 2003;27(2):153-158.

13. Bentrem D, Wilton A, Mazumdar M et al. The value of peritoneal cytology as a preoperative predictor in patients with gastric carcinoma undergoing a curative resection. Ann Surg Oncol. 2005;12(5):347-353

14. Saito $\mathrm{H}$, Kuroda $\mathrm{H}$, Matsunaga $\mathrm{T}$ et al. Prognostic indicators in node-negative advanced gastric cancer patients. J Surg Oncol. 2010;101(7):622-625.
15. Maehara $Y$, Hasuda S, Koga $T$ et al. Postoperative outcome and sites of recurrence in patients following curative resection of gastric cancer. Br J Surg. 2000;87(3):353-357.

16. Marrelli D, Roviello F, de Manzoni G et al. Different patterns of recurrence in gastric cancer depending on Lauren's histological type: longitudinal study. World J Surg. 2002;26(9):1160-1165.

17. Roviello F, Marrelli D, de Manzoni G et al. Prospective study of peritoneal recurrence after curative surgery for gastric cancer. Br J Surg. 2003;90(9):1113-1119.

18. Li H, Zhang SW, Liu J et al. Review of clinical investigation on recurrence of gastric cancer following curative resection. Chin Med J. 2012;125(8):1479-1495.

19. Sobin LH, Gospodarowicz MK, Wittekind Ch, International Union against Cancer. TNM: Classification of malignant tumors, 7th edition, 2009. Chichester, West Sussex, UK, Hoboken NJ: Wiley-Blackwell;2010:310.

20. Schwarz RE, Zagala-Nevarez K. Recurrence patterns after radical gastrectomy for gastric cancer: prognostic factors and implications for postoperative adjuvant therapy. Ann Surg Oncol. 2002;9(4):394-400.

21. Skoropad VY, Berdov BA. Influence of the morphological structure of stomach cancer on patterns of recurrence and metastasis. Questions Oncology. 2009;55(1):60-65.

22. La Torre M, Ferri M, Giovagnoli MR et al. Peritoneal wash cytology in gastric carcinoma. Prognostic significance and therapeutic consequences. Eur J Surg Oncol. 2010;36(10):982-986.

23. Nered SN. Surgical and combined treatment of gastric cancer with a high risk of implantation metastasis and resected gastric cancer: Authoref. of thesis on scientific degree dr. med. sciences. Moscow;2004:21 p.

24. D’Ugo D, Persiani R, Ojetti V et al. Prediction of lymph node metastasis in gastric cancer. The 8th International gastric cancer congress (IGCC): abstract book. - Krakow (Poland), 2009, 58.

25. Bando $\mathrm{E}$, Yonemura $\mathrm{Y}$, Takeshita $\mathrm{Y}$ et al. Intraoperative lavage for cytological examination in 1297 patients with gastric carcinoma. Am J Surg. 1999;178(3):256-262.

26. Kaibara N, Iitsuka Y, Kimura A et al. Relationship between the area of serosal invasion and prognosis in patients with gastric carcinoma. Cancer. 1987;60(1):136-139.

27. Ikeguchi M, Kondou A, Oka A et al. Effects of continuous hyperthermic peritoneal perfusion on prognosis of gastric cancer with serosal invasion. Eur J Surg. 1995;161:581-586.

28. Adachi Y, Yasuda K, Inomata M et al. Pathology and prognosis of gastric carcinoma. Well versus poorly differentiated type. Cancer. 2000;89(7):1418-1424.

29. Skoropad VY, Berdov BA. Clinico-morphological parallels in patients with gastric cancer. Questions Oncology. 2009;55(2):165-170.

30. Fukagawa T, Katai H, Saka M et al. Significance of lavage cytology in advanced gastric cancer patients. World J Surg. 2010;34(3):563-568.

31. Moriguchi S, Maehara Y, Korenaga D et al. Risk factors which predict pattern of recurrence after curative surgery for patients with advanced gastric cancer. Surg Oncol. 1992;1(5):341-346.

32. Japanese Gastric Cancer Association: Japanese classification of gastric cancer. - 2nd English ed. Gastric Cancer. 1998;1(1):10-24.

33. Nered SN, Klimenkov AA, Stilidi IS. Signet-ring cell gastric cancer: clinical and morphological aspects, the results of surgical treatment and prognosis. Questions Oncology. 2006;52(3):294-300.

34. Ohtsu A, Yoshida S, Saijo N. Disparities in gastric cancer chemotherapy between the East and West. J Clin Oncol. 2006;24(14): 2188-2196.

35. Hermans J, Bonenkamp JJ, Boon MC et al. Adjuvant therapy after curative resection for gastric cancer: meta-analysis of randomized trials. J Clin Oncol. 1993;11(8):1441-1447.

36. Janunger KG, Hafstrom L, Glimelius B. Chemotherapy in gastric cancer: a review and updated meta-analysis. Eur J Surg. 2002;168(11):597-608.

37. Sacuramoto $S$, Sasako M, Yamaguchi $T$ et al. Adjuvant chemotherapy for gastric cancer with S-1, an oral fluoropyrimidine. N Engl J Med. 2007;357(18):1810-1820. 
38. Fujitani K, Sasako M, Iwasaki Y et al. A phase II study of preoperative chemotherapy with S-1 and cisplatin followed by gastrectomy for clinically respectable type 4 and large type 3 gastric cancer: JCOG 0210. J Clin Oncol. 2007;25:4609 abstract. [2007 ASCO Meeting proceedings (post-meeting edition)].

39. Yoshikawa T, Omura K, Kobayashi O et al. A phase II study of preoperative chemotherapy with S-1 plus cisplatin followed by D2/ D3 gastrectomy for clinically serosa-positive gastric cancer (JACCRO GC-01 study). Eur J Surg Oncol. 2010;36(6):546-551.

40. Sugarbaker PH, Yonemura Y. Clinical pathway for the management of resectable gastric cancer with peritoneal seeding: best palliation with a ray of hope for cure. Oncology. 2000;58(2):96-107.

41. Chua TC. Progress in the combined modality management of peritoneal carcinomatosis. J Surg Oncol. 2010;102(7):728-729.

42. Weisberger AS, Levine B, Storaasli JP. Use of nitrogen mustard in treatment of serous effusions of neoplastic origin. J Am Med Assoc. 1955;159(18):1704-1707.

43. Kimura T, Koyama Y. Preventive chemotherapy after radical operations in stomach cancer. Adv Med Oncol. 1979;9:255-268.

44. Spratt JS, Adcock RA, Muskovin M et al. Clinical delivery system for intraperitoneal hyperthermic chemotherapy. Cancer Res. 1980;40(2): 256-260.

45. Dedrick RL. Theoretical and experimental bases of intraperitoneal chemotherapy. Semin Oncol. 1985;12(3):1-6.
46. De Roover A, Detroz B, Detry O et al. Adjuvant hyperthermic intraperitoneal peroperative chemotherapy (HIPEC) associated with curative surgery for locally advanced gastric carcinoma. An initial experience. Acta Chir Belg. 2006;106(3):297-301.

47. Fujimoto S, Takahashi M, Mutou T et al. Successful intraperitoneal hyperthermic chemoperfusion for the prevention of postoperative peritoneal recurrence in patients with advanced gastric carcinoma. Cancer. 1999;85(3):529-534.

48. Yonemura Y, de Aretxabala X, Fujimura T et al. Intraoperative chemohyperthermic peritoneal perfusion as an adjuvant to gastric cancer: final results of a randomized controlled study. Hepatogastroenterol. 2001;48(42):1776-1782.

49. Kim JY, Rhew CH, Kim KS et al. Controlled clinical trial for the gastric carcinoma patients underwent surgery plus IHCP. The 3th International gastric cancer congress (IGCC): abstract book - Seoul (Korea). 1999;106.

50. Xu DZ, Zhan YQ, Sun XW et al. Meta-analysis of intraperitoneal chemotherapy for gastric cancer. World J Gastroenterol. 2004;18:2727-2730.

51. Yan TD, Black D, Sugarbaker PH et al. A systematic review and meta-analysis of the randomized controlled trials on adjuvant intraperitoneal chemotherapy for respectable gastric cancer. Ann Surg Oncol. 2007;14(10):2702-2713. 